\title{
KNOWLEDGE MANAGEMENT IN COMPUTATIONAL CHEMISTRY: A LITERATURE REVIEW
}

\author{
Mr. Sunil Khilari ${ }^{1}$ and Dr. Sachin Kadam ${ }^{2}$
}

\begin{abstract}
As the twenty-first century unfolds, computational techniques are more expected to impact the fields of chemistry and its computational aspects. As chemical product design and development issues become more complex, chemists and scientists will need to have instant and reliable access to technical information along with advanced calculators and modeling tools. A powerful knowledge management system gives users those tools and technology to access critical information. More access to this information gives users a better understanding and that often leads to greater innovation. Without a knowledge management system, you will be much more limited to what you can do during the early stages of product development. Chemists are now able to investigate the properties of chemical structures at the molecular level. The aim of the research described in this paper is to in light on evolution of this field, present status, research directions based on various literature reviews. This paper also explores the gap, problems associated with knowledge and accordingly study objectives are framed and selected for Knowledge Management in Computational Chemistry study.
\end{abstract}

\section{ABOUT COMPUTATIONAL CHEMISTRY DOMAIN}

Chemists have been doing computations for centuries, but the field we know today as "computational chemistry" is a product of the digital age. Computational chemistry is a branch of chemistry that uses computer simulation to assist in solving chemical problems. It uses methods of theoretical chemistry, incorporated into efficient computer programs, to calculate the structures and properties of molecules and solids. However, more frequently the use of computers to make chemical predictions. Sometimes computational chemistry is used to predict new molecules or new reactions which are later investigated experimentally.

\section{EVOLUTION OF THE FIELD}

The discovery of sub-atomic particles such as the electron, proton and neutron sow the seeds of computation chemistry as an independent field of scientific inquiry. Specifically, researchers of the day debated competing theories concerning atom organization, and the mechanistic underpinnings of the forces mediating interactions between sub-atomic particles. Success of the quantum mechanical approach (over classical physics) in explaining the key observation that orbiting negatively-charged electrons do not spiral into the positively

\footnotetext{
${ }^{1}$ Bharati Vidyapeeth Deemed University Institute of Management \& Entrepreneurship Development, Pune

${ }^{2}$ Bharati Vidyapeeth Deemed University Institute of Management \& Entrepreneurship Development, Pune
} 
-charged nucleus ushered in the emerging field of electronic structure calculations, whose main goal at that time was to explain the emission spectra of various elements. Specifically, peaks present on the emission spectrum of elements (e.g., sodium and hydrogen) arise from the release or absorption of energy during transition of electrons between different energy levels. Realization that electrons, or more accurately, electron densities, are arranged in defined energy levels and spatial regions led to the proposal of the atomic and molecular orbital concepts. From a quantum mechanical perspective, these are regions where electrons of specific energies are located. This era was defined by the promulgation of many of the foundation concepts and tools of computation chemistry, where the theoretical tools of quantum mechanics illuminated spectroscopic observations, highlighting that theory lagged behind experiment. the second era of computation chemistry was also characterized by the emergence of many semi-empirical methods, where experiment data (usually from spectroscopy studies) were used in calibrating essential parameters in system models. These parameters describe key characteristics of atoms and could not be calculated from first principles in the pre -computing era. In addition, lack of computing power also constrained the types of systems studied to those involving single or few atoms. In the case of larger systems comprising more atoms, models exist to solve them approximately, but the simplifications used were unrealistic.

Computational chemists' daily work influences our understanding of the way the world works, helps manufacturers design more productive and efficient processes, characterizes new compounds and materials, and helps other researchers extract useful knowledge from mountains of data. Computational chemistry is also used to study the fundamental properties of atoms, molecules, and chemical reactions, using quantum mechanics and thermodynamics.

\section{PRESENT STATUS OF COMPUTATIONAL CHEMISTRY DOMAIN}

Building on the founding discoveries and theories in the history of quantum mechanics, the first theoretical calculations in chemistry were those of Walter Heitler and Fritz London in 1927. The books that were influential in the early development of computational quantum chemistry include Linus Pauling and E. Bright Wilson's 1935 Introduction to Quantum Mechanics - with Applications to Chemistry, Eyring, Walter and Kimball's 1944 Quantum Chemistry, Heitler's 1945 Elementary Wave Mechanics - with Applications to Quantum Chemistry, and later Coulson's 1952 textbook Valence, each of which served as primary references for chemists in the decades to follow.

Computational chemists may use simulations to identify sites on protein molecules that are most likely to bind a new drug molecule or create models of synthesis reactions to demonstrate the effects of kinetics and thermodynamics on the amount and kinds of products. They can also explore the basic physical processes underlying phenomena such as superconductivity, energy storage, corrosion, or phase changes.

The pharmaceutical industry, a major employer of computational chemists, has historically focused on the discovery and design of new small-molecular therapeutics. Recently, however, there is a trend to apply computational chemistry and cheminformatics (a field that combines laboratory data, chemical modeling, and information science methods) to process development, analytical chemistry, and biologics (medicinal products manufactured using or extracted from biological sources).

\section{RESEARCH DIRECTIONS IN COMPUTATIONAL CHEMISTRY DOMAIN}


As cheminformatics tools and computational modeling platforms develop, it becomes easier to define workflow tasks through graphically based workbench environments. A recent trend in reduced-order modeling and similar methods is enabling fairly powerful computational tools to be implemented on portable devices, including tablets and smart phones. This enables researchers to perform what-if calculations and try out various scenarios while they are in the plant or out in the field.

\section{Typical work duties include the following:}

- Develop computer models and simulations of chemical and biochemical processes and entities

- $\quad$ Perform and interpret statistical analysis of large datasets

- Create visual representations of reaction pathways, molecular interactions, or other phenomena

- $\quad$ Collaborate with laboratory researchers in industrial, nonprofit, government, or academic laboratories

Computational chemists, in contrast, may simply apply existing computer programs and methodologies to specific chemical questions.

Computational chemistry has two different aspects:

- $\quad$ Computational studies, used to find a starting point for a laboratory synthesis, or to assist in understanding experimental data, such as the position and source of spectroscopic peaks.

- Computational studies, used to predict the possibility of so far entirely unknown molecules or to explore reaction mechanisms not readily studied via experiments.

Thus, computational chemistry can assist the experimental chemist or it can challenge the experimental chemist to find entirely new chemical objects.

Several major areas may be distinguished within computational chemistry:

- $\quad$ The prediction of the molecular structure of molecules by the use of the simulation of forces, or more accurate quantum chemical methods, to find stationary points on the energy surface as the position of the nuclei is varied.

- $\quad$ Computational approaches to help in the efficient synthesis of compounds.

The researchers involved in computational chemistry to solve the problems using ICT tools and technologies. To enable this they need to well-define the involved problem.

- How does one determine if a computational problem is a new one?

- Is the problem well solved?

- Do similar or related problems exist?

- How can one be sure that a result is new?

- Who are the experts in this area, and is collaboration possible?

\section{LITERATURE REVIEW}

- T. Lee (1998) in his paper focuses that the Computational chemistry research is an established knowledge engineering process that that has allowed researchers to identify new or significant problems, to better understand existing approaches and experimental results, and to obtain new, effective and efficient solutions. While computational chemistry researchers regularly contribute to this knowledge base by proposing new problems and novel solutions, the processes currently used to share this knowledge are inefficient, resulting in unproductive overhead.

- T. Lee (1998) in his paper focuses that the Computational chemistry research is an established knowledge engineering process that that has allowed researchers to identify new or 
significant problems, to better understand existing approaches and experimental results, and to obtain new, effective and efficient solutions. While computational chemistry researchers regularly contribute to this knowledge base by proposing new problems and novel solutions, the processes currently used to share this knowledge are inefficient, resulting in unproductive overhead.

- Ikujiro Nonaka and Hirotaka Takeuchi, 1995, in their book The Knowledge Creating Company: How Japanese Companies Create the Dynamics of Innovation, presented the following model of how tacit and explicit knowledge interact in the knowledge creation process.

\section{RESEARCH GAP}

The literature review revealed knowledge management as the major concern in computational chemistry domain.

A knowledge management framework will assist a researcher from computational chemistry domain in survey efforts by providing an infrastructure for organizing and visualizing intellectual landscapes.

Similar knowledge management frameworks been developed in other domains of scientific computations like computational aerodynamics and fluid dynamics. Therefore this research proposes to develop such a framework for computational chemistry domain.

- The answers to these questions will immensely help a researcher from computational chemistry domain. A knowledge management framework will enable this.

- Such a framework will assist researchers in their survey efforts by providing an infrastructure for organizing and visualizing intellectual landscapes. Also its collaboration perspective will enable a researcher to contribute feedback or new insights to a shared knowledge structure.

Computational chemists in academic environments often teach courses or provide individualized instruction on using various types of software or data analysis. At national laboratories, they may train visiting users, and they may perform their own research. Customer service computational chemists may travel to their customers' laboratories to provide them with training or technical assistance.

\section{TOPIC SELECTED FOR RESEARCH}

This research proposes to develop a knowledge management framework based upon a welldefined ontology in computational chemistry domain and this framework will be implemented using open source technologies and then evaluated.

\section{OBJECTIVES FOR THE STUDY}

1. Study the formalized problem space involved in computational chemistry domain.

2. Analyze formalized problem space to formulate corresponding solution space.

3. Analyze solution space to formulate implementation space.

4. Generate ontology for computational chemistry through a holistic analysis of problem, solution and implementation spaces.

\section{HYPOTHESIS}

H1: Existence of wide gap between knowledge management in computational Chemistry domain and present expectations.

H2: Reaction Mechanism is greater computational problem than molecular structure 
H3: Algorithmic solution space is better tools for solving computational problem than physical model

\section{RESEARCH METHODOLOGY}

This research will follow the Survey plus Design and Creation research methodology.

- $\quad$ Survey based research for design of ontology

- Design \& creation research methodology for developing KM framework based on ontology

The design and creation research strategy for this research will focus on collaborative knowledge management architecture for computational chemistry problem solving. The theoretical population to collect the primary data for this research will consist of all the chemical manufacturing companies, pharmaceutical companies, medical shops, nursing, pharmacy institutions, s/w. developers and customers of chemical software. The accessible population will be considered from in and around Pune region.

\begin{tabular}{|l|l|}
\hline Particulars & Nos. \\
\hline $\begin{array}{l}\text { Research paper published in } \\
\text { international journals }\end{array}$ & $\mathbf{2}$ \\
\hline $\begin{array}{l}\text { Research paper presented in National } \\
\text { Conference }\end{array}$ & $\mathbf{1}$ \\
\hline $\begin{array}{l}\text { Workshop @ IIT,Bombay on Open } \\
\text { Source Chemistry software }\end{array}$ & $\mathbf{1}$ \\
\hline $\begin{array}{l}\text { Books referred } \\
\text { International Journals-Research Papers } \\
\text { referred }\end{array}$ & $\mathbf{4 3}$ \\
\hline $\begin{array}{l}\text { National Journals-Research Papers } \\
\text { referred }\end{array}$ & $\mathbf{5}$ \\
\hline $\begin{array}{l}\text { International Conference Papers } \\
\text { Referred }\end{array}$ & $\mathbf{1 0}$ \\
\hline Websites visited & $\mathbf{1 6}$ \\
\hline PHD Thesis Refereed & $\mathbf{8}$ \\
\hline
\end{tabular}



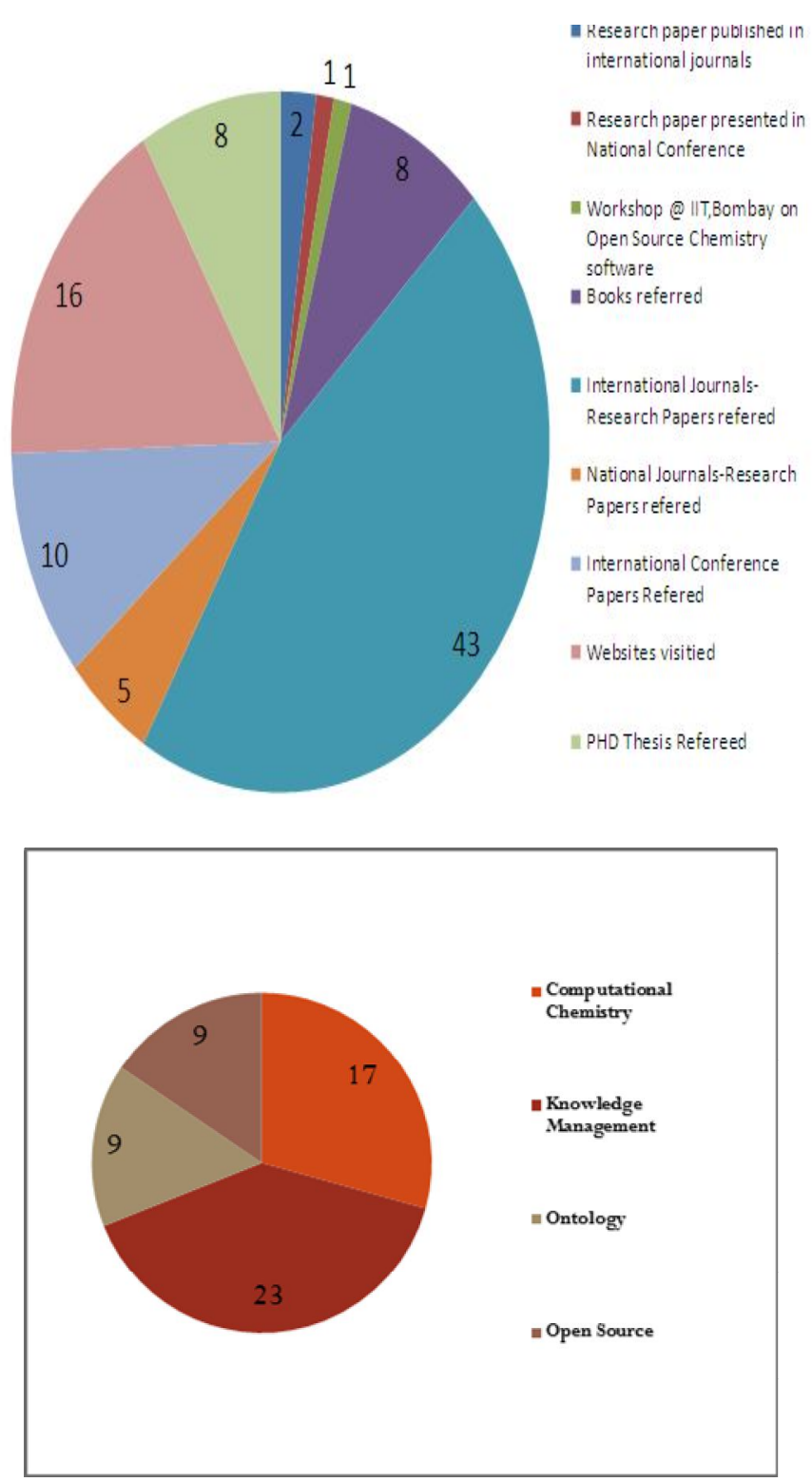

\section{BENEFIT OF THIS STUDY}

\section{Customers of Chemical Software's}

$\checkmark \quad$ Storing and searching for data on chemical entities

$\checkmark \quad$ Readily accessible database of computational chemistry results

$\checkmark \quad$ Problem specific information on the successful application of computational tools.

$\checkmark \quad$ Software Application usage for accuracy

$\checkmark \quad$ Powerful software tools can support creativity

$\checkmark \quad$ Increased knowledge on problem, solution and implementation spaces

$\checkmark \quad$ Reusing ideas, documents and expertise

\section{IT Developers}

$\checkmark$

Get more understanding of the chemical software development process

Help to deriving meaningful knowledge out of data

Convert tacit chemical knowledge to explicit form chemical knowledge 
$\checkmark \quad$ Check algorithmic solutions are available or if there is need for new computational problem object.

$\checkmark \quad$ Help to build powerful chemical software knowledge-base in the future.

\section{Chemical Manufacturers}

$\checkmark \quad$ Identifying correlations between chemical structures and properties

$\checkmark \quad$ To design molecules that interact in specific ways with other molecules

$\checkmark \quad$ Understand transformation on categories

$\checkmark \quad$ To search a problem space to see if a computational problem is well-defined

$\checkmark \quad$ To make computational chemistry more accessible in terms of the entry point in chemical domain.

\section{Pharmaceutical Companies}

$\checkmark \quad$ Help in the efficient synthesis of compounds

$\checkmark \quad$ Expert help in choosing options needed to run computations

$\checkmark \quad$ New knowledge is built on previous knowledge

$\checkmark \quad$ Effective knowledge management supports advancement of product and process understanding as knowledge flows in computational chemistry

\section{CONCLUSION}

Computational problem solving researchers can search a problem space to see if a computational problem is well-defined, if computational chemistry solutions are available, or if there is a need for a new computational problem object and the potential for a collaborative effort to create it. The problem space, which corresponds to well-defined computational problems, is the heart of the computational problem solving research domain. The solution space, which corresponds to Existing algorithmic solutions, provides an up-to-date theoretical view of problem solving efforts made by computational problem solving researchers.

\section{REFERENCES}

[1]. Smith, S. J.; Sutcliffe, B. T. (1997). "The development of Computational Chemistry in the United Kingdom". Reviews in Computational Chemistry. 10: 271-316.

[2]. Schaefer, Henry F. III (1972). The electronic structure of atoms and molecules. Reading, Massachusetts: Addison-Wesley Publishing Co. p. 146.

[3]. Boys, S. F.; Cook, G. B.; Reeves, C. M.; Shavitt, I. (1956). "Automatic fundamental calculations of molecular structure". Nature. 178 (2): 1207. Bibcode:1956Natur.178.1207B. doi:10.1038/1781207a0.

[4]. Richards, W. G.; Walker, T. E. H.; Hinkley R. K. (1971). A bibliography of ab initio molecular wave functions. Oxford: Clarendon Press.

[5]. Preuss, H. (1968). "DasSCF-MO-P(LCGO)-Verfahren und seine Varianten". International Journal of Quantum Chemistry. 2 (5): 651. Bibcode:1968IJQC....2..651P. doi:10.1002/qua.560020506.

[6]. Buenker, R. J.; Peyerimhoff, S. D. (1969). "Ab initio SCF calculations for azulene and naphthalene". Chemical Physics Letters. 3: 37. Bibcode:1969CPL.....3...37B. doi:10.1016/0009-2614(69)80014-X.

[7]. Schaefer, Henry F. III (1984). Quantum Chemistry. Oxford: Clarendon Press.

[8]. Streitwieser, A.; Brauman, J. I.; Coulson, C. A. (1965). Supplementary Tables of Molecular Orbital Calculations. Oxford: Pergamon Press.

[9]. Pople, John A.; Beveridge, David L. (1970). Approximate Molecular Orbital Theory. New York: McGraw Hill.

[10]. Allinger, Norman (1977). "Conformational analysis. 130. MM2. A hydrocarbon force field utilizing V1 and V2 torsional terms". Journal of the American Chemical Society. 99 (25): 8127-8134. doi:10.1021/ja00467a001.

[11]. Fernbach, Sidney; Taub, Abraham Haskell (1970). Computers and Their Role in the Physical Sciences. Routledge. ISBN 0-677-14030-4. 